This document is published in:

Journal of Oral and Maxillofacial Surgery, Vol. 70, no 10, pp. 2453-2458

DOI: http://dx.doi.org/10.1016/j.joms.2011.10.028

(C) 2012 American Association of Oral and Maxillofacial Surgeons. 


\title{
Use of Autologous Skin Equivalents With Artificial Dermal Matrix (Integra) in Donor Site Coverage in Radial Forearm Free Flaps: Preliminary Cases
}

\author{
Ignacio Peña, MD, DDS, PhD, * \\ Lucas de Villalaín, MD, DDS, PhD,† Eva García, PhD, $\neq$ \\ Luis Manuel Junquera, MD, DDS, PhD, $\int$ and \\ Juan Carlos de Vicente, $M D, D D S, P h D \|$
}

\begin{abstract}
Purpose: The radial forearm flap is one of the most commonly used methods for intraoral reconstruction in oral carcinoma surgery. One of its disadvantages is the residual functional and unaesthetic defect in the donor site. The objective of this report is to describe preliminary cases of a novel method to cover such donor sites based on the use of autologous skin equivalents (ASEs) and an artificial dermal matrix (Integra, Prim, Barcelona, Spain).
\end{abstract}

Materials and Methods: The donor sites of 2 patients were treated with the artificial dermal matrix after raising a radial forearm flap. A skin biopsy and a blood sample were taken to construct an ASE. After 3 weeks, the ASE was applied over the dermal template and left to heal. The functional and esthetic results were recorded.

Results: Good functional and esthetic results were achieved, with correct wrist motility, although a natural skin color could not be achieved. Neither the Integra nor the ASE was rejected. Total wound coverage was achieved at 4 months, and completely normal skin was observed at 6 months.

Conclusions: This technique of combining an artificial dermal matrix with an ASE could be an alternative method to cover the donor sites of radial forearm flaps.

The radial forearm flap originally described by Yang et $\mathrm{al}^{1}{ }^{1}$ and Soutar et $\mathrm{al}^{2}$ popularized its use in oral reconstruction. Its thickness, pliability, good pedicle, and easy extraction, and the possibility of a simultaneous 2-team approach made it a good choice for oral reconstruction. One of its disadvantages, however, was morbidity to the donor site.

Different methods have been used to solve this problem, such as full- or partial-thickness skin grafts, ${ }^{3}$ an artificial dermal matrix with ultrathin skin grafts, ${ }^{4}$ cubital rotational flaps, ${ }^{5}$ or tissue ex- panders before surgery. ${ }^{6}$ However, none is free from complications, such as the partial or total loss of the graft, chronic lymphedema, neurosensory alterations, tendon exposure, and unaesthetic results. In addition, a second donor site is needed.

Satisfactory clinical results have been achieved with dermal templates (Integra) in combination with ultrathin skin grafts. ${ }^{7}$

The objective of this report is to describe the preliminary cases of a novel method using an artificial dermal matrix with autologous skin equivalents
Received from the Department of Oral and Maxillofacial Surgery, Central University Hospital of Asturias, Oviedo, Asturias, Spain.

*Staff.

†Staff.

¥Tissue Engineer, Community Center of Blood and Tissues of the Princedom of Asturias, Oviedo, Asturias, Spain.

§Staff.

||Department Head.
Address correspondence and reprint requests to Dr Peña: Department of Oral and Maxillofacial Surgery, Central University Hospital of Asturias, C/Celestino Villamil s/n, 33006 Oviedo, Asturias, Spain; e-mail: napegomaxilo@gmail.com 
(ASEs) ${ }^{8,9}$ for the treatment of donor sites of radial forearm flaps.

\section{Materials and Method}

\section{ENGINEERING ASE}

Skin biopsies were obtained from the flap or the tracheostomy. All patients received detailed oral and written information, and an informed consent approved by the local institutional review board was obtained. Subsequently, the biopsies were processed as previously described. ${ }^{8,9}$ Briefly, the samples were divided into small fragments and subjected to 4 washes with phosphate buffered saline $5 \mathrm{~mL}$ containing trypsin and ethylenediaminetetraacetic acid (Gibco, Paisley, Invitrogen, UK) at concentrations of $0.05 \%$ and $0.02 \%$, respectively.
The resulting cell pellet was used to obtain the primary keratinocyte culture. The remaining tissue fragments were subjected to a fifth wash with collagenase (Sigma, Madrid, Spain) and Dulbecco's modified Eagle's medium (DMEM) at a concentration of $2 \mathrm{mg} / \mathrm{mL}$ to obtain a cell pellet for use in the primary fibroblast culture.

The keratinocyte growth medium was labeled as QN [DMEM (Gibco, Invitrogen)] and Ham F-12 (Gibco, Invitrogen) at a ratio of $3: 1$ plus $10 \%$ fetal bovine serum (Gibco, Victoria, Invitrogen, Australia) plus insulin ( $5 \mu \mathrm{g} / \mathrm{ml}$; Sigma) plus cholera toxin (8 ng/mL; Sigma) plus adenine $(24 \mathrm{mg} / \mathrm{mL}$; Sigma) plus triiodothyronine $(1.3 \mathrm{ng} / \mathrm{mL}$; Sigma) plus hydrocortisone $(0.4 \mu \mathrm{g} / \mathrm{mL}$; Sigma) and required the use of lethally irradiated $3 \mathrm{~T} 3$ cells as a feeder layer. For the cultivation of fibroblasts, DMEM supple-

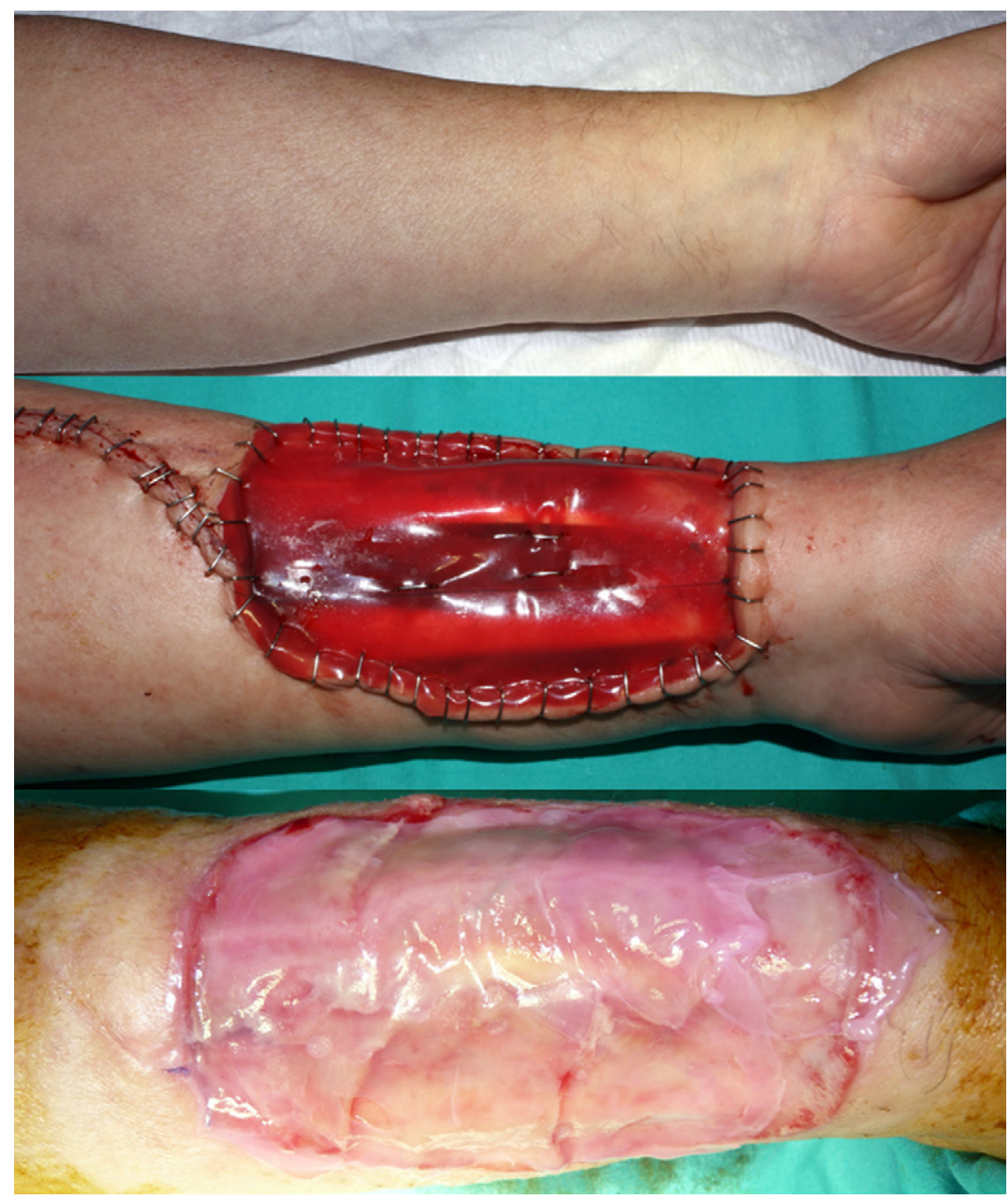

FIGURE 1. Top, Donor site before raising the flap. Middle, Bilayer artificial dermal matrix sheets are placed. Bottom, Grafting of an autologous skin equivalent over the new dermis. The autologous skin equivalent was easy to handle and had a thickness close to that of an ultrathin skin graft. 


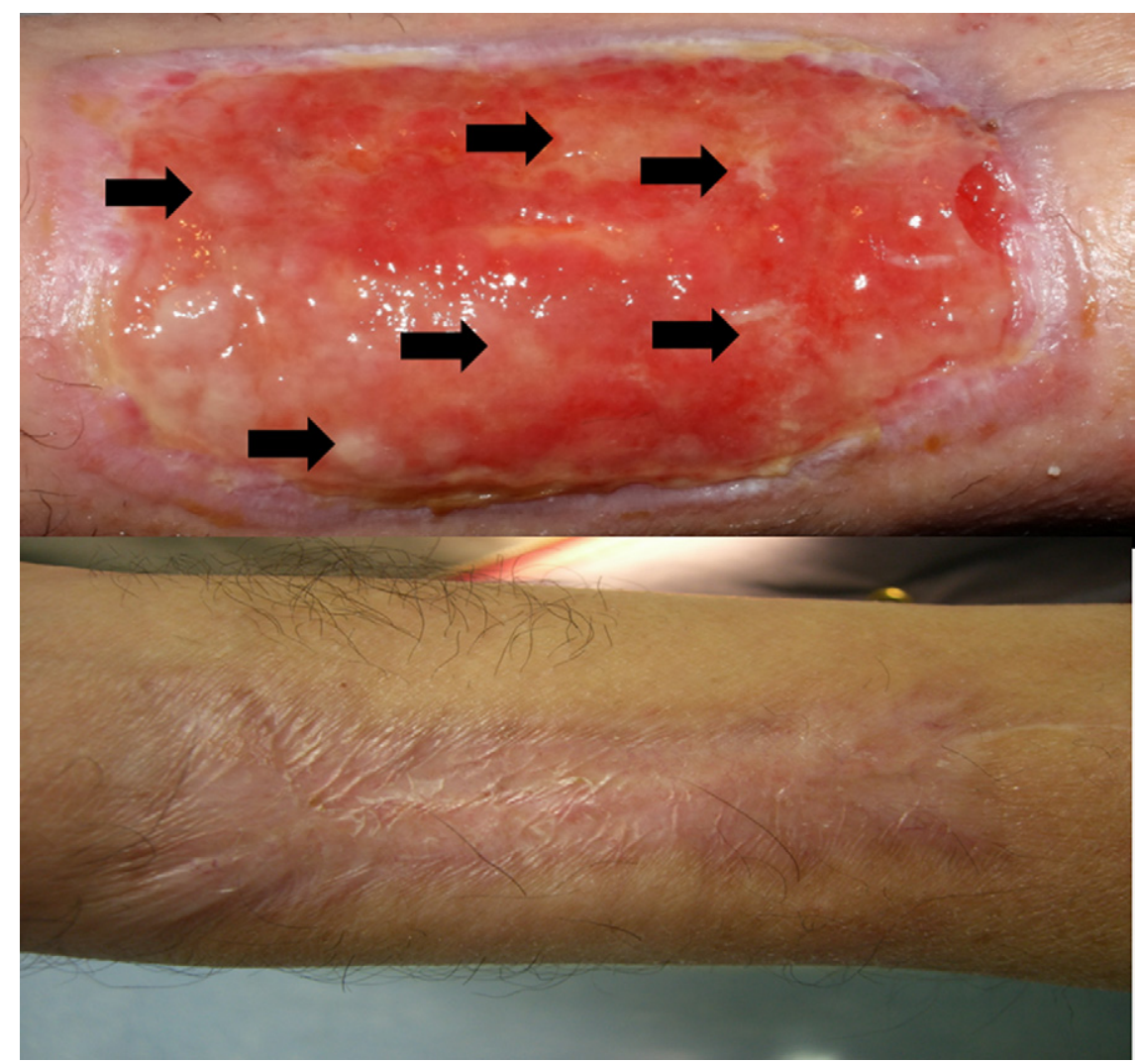

FIGURE 2. First case. Top, One week after grafting of the autologous skin equivalent, there are areas where clusters of mature cells seem to be developing (arrows). Bottom, Complete healing was achieved after 6 months.

mented with $10 \%$ fetal bovine serum was used. After 3 days of culture, epidermal growth factor (10 ng/mL; Austral Biologicals, San Ramon, CA) was added to the keratinocyte culture. When cells reached $90 \%$ confluence, a first pass was made into T-25 flasks for keratinocytes and into T-12.5 flasks for fibroblasts. The time until confluence for the first-pass keratinocytes was 7 to 8 days. During that period, a second pass was needed for fibroblasts into the T-75 flasks. Fibrin glue obtained from each patient's blood sample was used for scaffolding. To construct a $75-\mathrm{cm}^{2}$ ASE, plasma $12 \mathrm{~mL}$, fibroblasts $66 \times 10^{3}, 1 \%$ calcium chloride in saline serum 2.1 $\mathrm{mL}$, tranexamic acid $210 \mu \mathrm{L}$ (FIDES Ecofarma, Almacera, Spain), and saline serum were used. The mixture was then allowed to solidify at $37^{\circ} \mathrm{C}$ for 30 to 60 minutes with QN medium $4.7 \mathrm{~mL}$. After that period, keratinocytes were seeded. Fibroblasts were from the second pass and keratinocytes were from the first pass (one third of the total amount of the T-flask cells). The remaining cells were frozen as previously described. ${ }^{8}$ From a primary culture, 3 ASEs of $75 \mathrm{~cm}^{2}$ each were obtained. To avoid keratinocyte differentiation, the submerged method was used. ASE handling became easier by following the method described by Meana et al. ${ }^{10}$

\section{CASES AND SURGICAL PROCEDURES}

Two cases of oral epidermoid carcinoma were selected. A radial forearm flap was used for reconstruction in these cases. In the first case, the flap measured $9 \times 7 \mathrm{~cm}$; in the second case, a $10-\times$ $7-\mathrm{cm}$ free flap was used. During surgery, a skin biopsy was taken from the tracheostomy (first case) or from the free flap (second case). In addition, a blood sample of $27 \mathrm{~mL}$ was taken. In the first case, a $10-\times 12.5-\mathrm{cm}$ bilayer artificial dermal matrix (Integra, Prim, Barcelona, Spain) sheet was used; in the second case, $25-\mathrm{cm} \times 5$-cm bilayer sheets were applied.

The required periods before ASE grafting were 4 weeks in the first case and 3 weeks in the second case. An ASE was then grafted over the new dermis without the need for anesthesia. Sutures were not needed; instead, homogeneous compression with gauzes and bandage was applied for 7 days (Fig 1). 


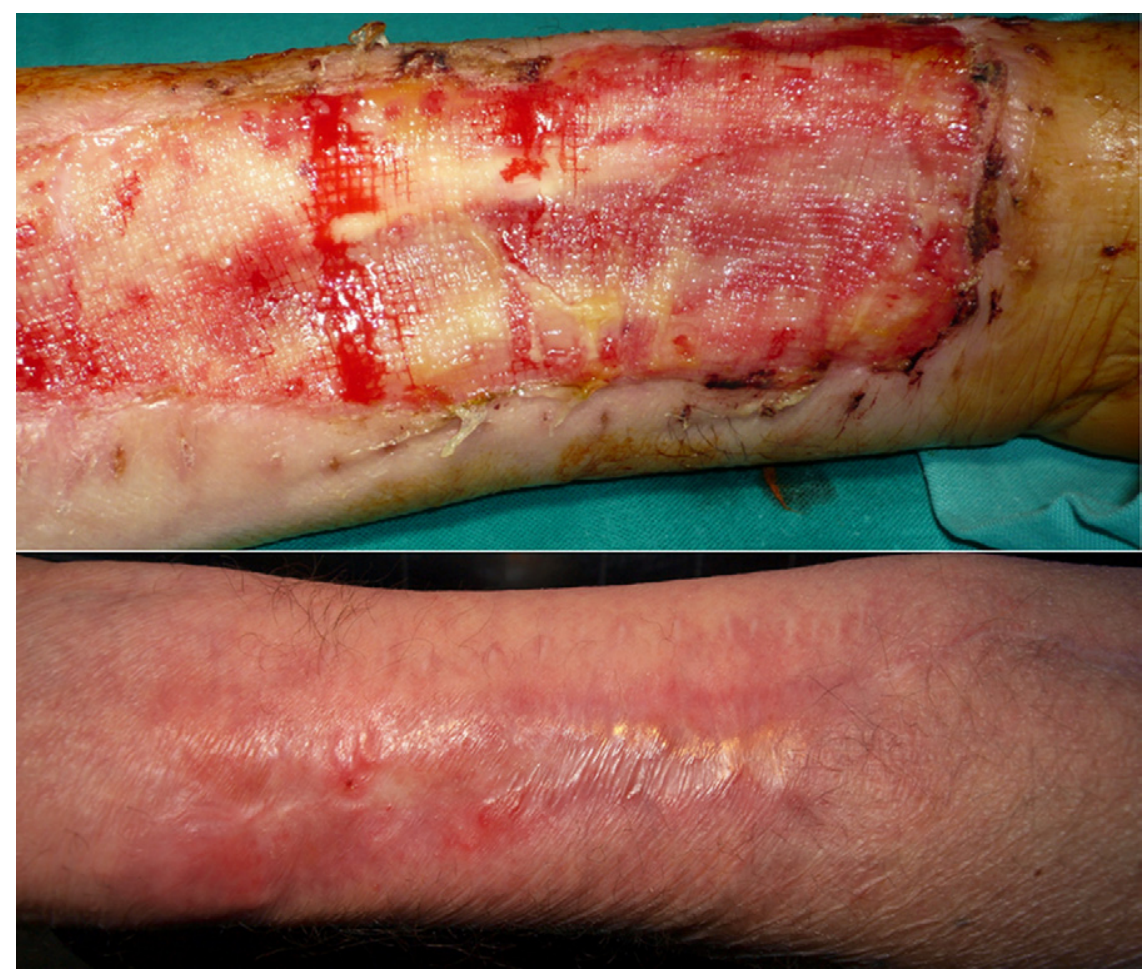

FIGURE 3. Second case. Top, Three weeks after grafting of the autologous skin equivalent, the appearance of the graft is closer to that of normal skin when compared with the 1-week stage. Bottom, Complete healing was achieved after 4 months.

\section{Results}

After 1 week, all ASEs successfully took hold. During the second week, the wound area became progressively smaller. Macroscopically, there were spots exhibiting more epidermal differentiation surrounded by the ASE; the authors believe stratification and differentiation were taking place in those areas. Vascularization also increased. After 2 months, the first case required a second ASE because a central defect persisted without epidermal coverage. A new ASE was designed using frozen cells from the patient, and after 4 months complete healing was achieved. In the second case, complete wound healing was accomplished at 4 months without the need for a new ASE (Figs 2, 3).

Appropriate functional and esthetic results were achieved, as was a correct mobilization of the wrist. One disadvantage observed was a color alteration of the regenerated skin, but this was not considered relevant by the patients (Fig 4).

No pain, infections, or other complications were recorded. Patients also showed normal cutaneous sensitivity in the grafted area.

\section{Discussion}

The method of choice to close donor sites after obtaining a radial forearm flap is primary closure with sutures ${ }^{11}$ or by a local cubital rotational flap. The cubital flap limits the width of the donor area to $6 \mathrm{~cm}^{5}$ and may present disadvantages such as chronic lymphedema and denervation of the volar forearm. ${ }^{4}$

In cases in which the width is greater than $6 \mathrm{~cm}$, the classic method to cover the donor site involves the use of a split-thickness skin graft, which causes major issues, such as pain and sensibility alterations in the graft donor site, tendinous exposure, and poor functional and esthetic results. ${ }^{4}$ To overcome these problems, different techniques have been used, such as the combination of ultrathin skin grafts with a de-epidermized acellular dermis ${ }^{4}$ or an artificial dermal matrix. ${ }^{7}$ The objective is to not only provide coverage, but also restore full functioning and appearance by adding epidermis and dermis to aid in wound healing.

Under this premise, the authors decided to use bilayer artificial dermal matrix sheets to obtain a new dermis, a fundamental component to obtaining good function and appearance in wound healing, and to combine it with the authors' experience in tissue engineering. ${ }^{8,9}$ By following this method, neither a second surgery nor a second donor site was needed. 


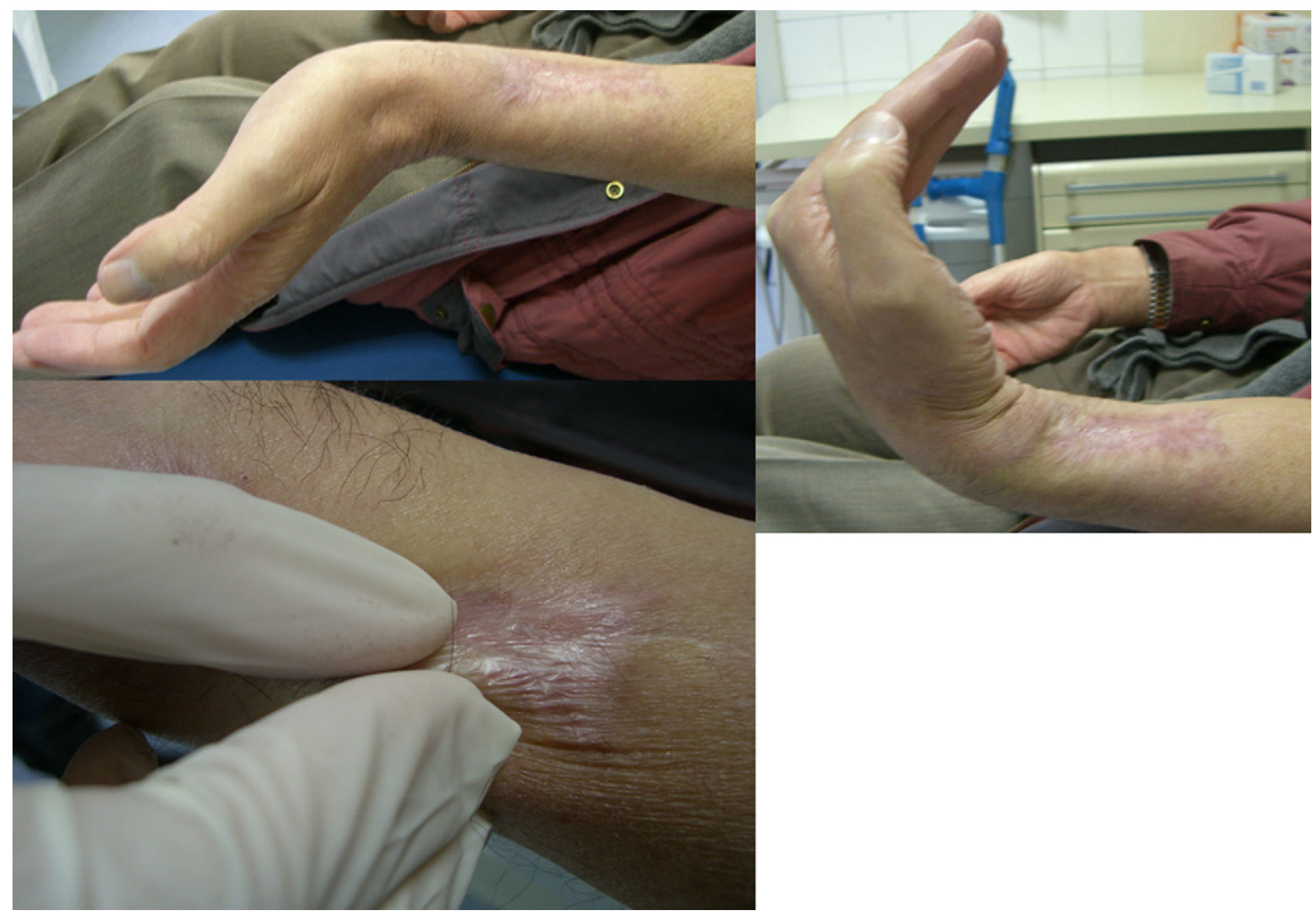

FIGURE 4. Top left and right, Functional assessment was satisfactory, with a correct mobility of the wrist. Bottom left, Optimal skin flexibility was achieved from the dermal regeneration compared with outcomes when using split-thickness skin grafts alone.

The ASE management was similar to that observed with split-thickness skin grafts. Adaptation to the forearm defects was easy because of its scaffold of fibrin glue; hence, sutures were not needed. In that sense, the procedure could proceed without anesthesia. Another advantage was cells could be frozen and used to construct another ASE, if needed.

The functional and esthetic outcomes were comparable to those observed by other investigators, ${ }^{4,7}$ with good wrist motility and skin elasticity. Appearance was good except for the skin color, a problem related to the use of the artificial dermal matrix described previously by others. ${ }^{7}$ The skin sensibility was normal.

A disadvantage worth mentioning could be the eventual necessity of a second ASE, as in the first case. Although patients did not report any discomfort, this requirement could be attributable to the fact that stem cells of the ASE mature early or that the cell volume in the ASE was not adequate to achieve complete healing, a hypothesis that needs to be studied in future researches.
The use of an artificial dermal matrix with an ASE in the donor site of a radial forearm flap is a novel approach, with less morbidity compared with other techniques. Even if a second ASE is required at a later stage, the low morbidity, the lack of need of a second donor site and additional surgery, the optimal functional outcome, and the acceptable esthetic results make this technique promising. Nevertheless, further studies with a larger number of patients are needed to validate the present findings.

\section{References}

1. Yang G, Chen B, Gao Y, et al: Forearm free skin flap transplantation. Natl Med J China 61:139, 1981

2. Soutar DS, Scheker LR, Tanner NSB, et al: The radial forearm flap: A versatile method for intra-oral reconstruction. Br J Plast Surg 36:1, 1983

3. González-García R, Ruiz Laza L, Manzano D, et al: Combined local triangular full-thickness skin graft for the closure of the radial forearm free flap donor site: A new technique. J Oral Maxillofac Surg 67:1562, 2009

4. Rowe NM, Morris L, Delacure MD: Acellullar dermal composite allografts for reconstruction of the radial forearm donor site. Ann Plast Surg 57:305, 2006 
5. Ahn HC, Choi MSS, Hwang WJ, et al: The transverse radial artery forearm flap. Plast Reconstr Surg 119:2153, 2007

6. Hallock GG: Refinement of the radial forearm flap donor site using skin expansion. Plast Reconstr Surg 81:21, 1988

7. Gravvanis AI, Dimosthenis AT, Iconomou T, et al: The use of integra artificial dermis to minimize donor-site morbidity after suprafascial dissection of the radial forearm flap. Microsurgery 27:583, 2007

8. Llames SG, Del Rio M, Larcher F, et al: Human plasma as a dermal scaffold for the generation of a completely autologous bioengineered skin. Transplantation 77:350, 2004
9. Peña I, Junquera LM, Meana A, et al: In vitro engineering of complete autologous oral mucosa equivalents: Characterization of a novel scaffold. J Periodont Res 45:375, 2010

10. Meana A, Iglesias J, Madrigal B, et al: Use of cyanoacrylate glue to prepare cultured keratinocyte sheets for grafting. Burns 23:645, 1997

11. Moazzam A, Gordon DJ: Cross suturing as an aid to wound closure: A prospective randomised trial using the forearm flap donor site as a model. Br J Plast Surg 56:695, 2003 